Article

\title{
The Global Jet Structure of the Archetypical Quasar 3C 273
}

\author{
Kazunori Akiyama ${ }^{1,2,3, *}$, Keiichi Asada ${ }^{4}$, Vincent L. Fish ${ }^{2}$ (D), Masanori Nakamura ${ }^{4}$, \\ Kazuhiro Hada ${ }^{3}$ (D), Hiroshi Nagai ${ }^{3}$ and Colin J. Lonsdale ${ }^{2}$ \\ 1 National Radio Astronomy Observatory, 520 Edgemont Rd, Charlottesville, VA 22903, USA \\ 2 Massachusetts Institute of Technology, Haystack Observatory, 99 Millstone Rd, Westford, \\ MA 01886, USA; vfish@haystack.mit.edu (V.L.F.); cjl@haystack.mit.edu (C.J.L.) \\ 3 National Astronomical Observatory of Japan, 2-21-1 Osawa, Mitaka, Tokyo 181-8588, Japan; \\ kazuhiro.hada@nao.ac.jp (K.H.); hiroshi.nagai@nao.ac.jp (H.N.) \\ 4 Institute of Astronomy and Astrophysics, Academia Sinica, P.O. Box 23-141, Taipei 10617, Taiwan; \\ asada@asiaa.sinica.edu.tw (K.A.); nakamura@asiaa.sinica.edu.tw (M.N.) \\ * Correspondence: kakiyama@mit.edu
}

Received: 16 September 2017; Accepted: 8 January 2018; Published: 24 January 2018

\begin{abstract}
A key question in the formation of the relativistic jets in active galactic nuclei (AGNs) is the collimation process of their energetic plasma flow launched from the central supermassive black hole (SMBH). Recent observations of nearby low-luminosity radio galaxies exhibit a clear picture of parabolic collimation inside the Bondi accretion radius. On the other hand, little is known of the observational properties of jet collimation in more luminous quasars, where the accretion flow may be significantly different due to much higher accretion rates. In this paper, we present preliminary results of multi-frequency observations of the archetypal quasar 3C 273 with the Very Long Baseline Array (VLBA) at 1.4, 15, and 43 GHz, and Multi-Element Radio Linked Interferometer Network (MERLIN) at 1.6 GHz. The observations provide a detailed view of the transverse structure resolved on a broad range of spatial scales from sub-parsec to kilo parsecs, allowing us to profile the jet width as a function of the distance from the core for the first time in jets of bright quasars. We discovered a transition from a parabolic stream to a conical stream, which has been seen in much lower-luminosity radio galaxies. The similarity in the profile to the much lower-powered radio galaxy M87 suggests the universality of jet collimation among AGNs with different accretion rates.
\end{abstract}

Keywords: galaxies: active; galaxies: individual (3C 273); galaxies: jets; radio continuum: galaxies; techniques: high angular resolution; techniques: interferometric

\section{Introduction}

The relativistic jets in active galactic nuclei (AGNs) are some of the most energetic persistent plasma outflows in the Universe, which may affect the (co-)evolution of their host galaxies and the central super-massive black holes (SMBHs) through kinetic feedback. One of key processes in their formation is collimation, which focuses their relativistic flow into a narrow angle and allows it to propagate its enormous energy to the inter-stellar/galactic medium generally much larger than the central accretion flow (see Section 3 for more details on theory work).

The most direct observational approach to study this issue is to constrain the transverse structure of the jet along its length in order to quantify how and where the jet collimates. In recent years, the transverse structure of the jet has been intensively studied for low-luminosity radio galaxies that are thought to be powered by geometrically thick radiatively inefficient accretion flows [1]. The radio galaxy M87 shows a clear picture of its jet exhibiting a parabolic profile, implying an active collimation process [2-5]. This parabolic profile persists up to the peculiar stationary knot HST-1 near the Bondi 
accretion radius of $\sim 10^{5}$ Schwarzschild radii $\left(R_{s}\right)$, then appears to transition to a conical profile without collimation beyond that point. A very similar transitional profile has recently been obtained in the radio galaxy NGC 6251 [6], and parabolic collimation inside the Bondi accretion radius has also been confirmed in the nearby radio galaxies 3C 84 [7] and Cyg A [8]. On the other hand, little is known for high-powered AGNs, which are driven by accretion flows with much higher accretion rates.

3C 273 is one of the brightest and most well-known AGNs, since it is historically the firstdiscovered quasar. Its proximity as a radio-loud quasar $(\mathrm{z}=0.158)$ and its extraordinarily massive black hole $\left(6.59_{-0.90}^{+1.67} \times 10^{9} M_{\odot}\right.$; [9] $)$ provide the largest angular diameter of the SMBH among the known radio-loud quasars $\left(1 \mathrm{mas}=2.7 \mathrm{pc}=4.8 \times 10^{3} R_{s}\right)$. The jet structure has been clearly resolved transversely in a broad spatial range from milliarcsecond to arcsecond angular scales (e.g., $[10,11])$. This makes 3C 273 an ideal source to extend previous efforts on the streamline analysis to more powerful quasar jets.

In this paper, we report our preliminary results of multi-frequency observations of 3C 273 with the Very Long Baseline Array (VLBA) at 1.4/15/43 GHz and the Multi-Element Radio Linked Interferometer Network (MERLIN) at 1.6 GHz. We present the global jet structure of the quasar 3C 273 on milliarcsecond to arcsecond scales. We adopted the black hole mass of $6.59_{-1.00}^{+1.68} \times 10^{9} \mathrm{M}_{\odot}[9]$ and viewing angle of $3.8^{\circ}$, which is its minimum limit for the viewing angle of $3 \mathrm{C} 273\left(3.8^{\circ}-7.2^{\circ}\right.$; [12]).

\section{Observational Data and Analysis}

\subsection{Observational Data}

VLBA $43 \mathrm{GHz}$ data-We used VLBA $43 \mathrm{GHz}$ data at 50 epochs from 2007 to 2016 obtained with the monthly monitoring program of the Boston University Blazar Group. We used the CLEAN models and self-calibrated visibility data, available at their website ${ }^{1}$. The typical angular resolution of observations was 0.38 mas $\times 0.15$ mas with a position angle (PA) of $-10^{\circ}$ for the uniform weighted image. We restored the uniform-weighted CLEAN image with a circular Gaussian beam of 0.38 mas in DIFMAP [13], corresponding to the typical major-axis size of the synthesized beam.

VLBA $15 \mathrm{GHz}$ data-We used VLBA $15 \mathrm{GHz}$ data of the MOJAVE program (Monitoring Of Jets in Active galactic nuclei with VLBA Experiments; [14]) at 84 epochs from 1995 to 2016 . We used self-calibrated visibility data available in their website ${ }^{2}$. The typical angular resolution of observations was 1.00 mas $\times 0.46$ mas with a PA of $-3^{\circ}$ for the uniform-weighted image. We note that there was no observation in 2011. We produced the uniform-weighted CLEAN image restored with a circular Gaussian beam of 1 mas in DIFMAP.

VLBA 1.4 GHz data-We selected eight epochs of archival VLBA $1.4 \mathrm{GHz}$ data from 2008 to 2011 with good hour-angle coverage. Data were initially reduced in the Astronomical Imaging Processing System (AIPS) ${ }^{3}$ of the National Radio Astronomy Observatory (NRAO) in the standard manner, and imaged in DIFMAP with self-calibration. We produced uniform-weighted CLEAN images restored with a circular Gaussian beam of 9 mas in DIFMAP, corresponding to the major-axis size of the mean synthesized beam of $9 \times 3.5$ mas at a position angle of $-5^{\circ}$.

MERLIN 1.6 GHz data-We reduced archival MERLIN data observed in April 1993 at $1.6 \mathrm{GHz}$. Data were initially reduced in AIPS in the same manner as in Bahcall et al. [10], and imaged in DIFMAP using the central $1 \mathrm{MHz}$ channel following Bahcall et al. [10] to avoid bandpass smearing effects. We produced the natural-weighted CLEAN image, restored with the major-axis size of the synthesized beam of $256 \times 151$ mas at a position angle of $24^{\circ}$.

\footnotetext{
https://www.bu.edu/blazars/research.html

http://www.physics.purdue.edu/astro/MOJAVE/

http://www.aips.nrao.edu/
} 


\subsection{Stream Line Analysis}

3C 273 has a mostly straight streamline on arcsecond scales traced with MERLIN, similar to the sub-arcsecond and arcsecond-scale structure of M87 [2]. We followed a method adopted in Asada and Nakamura [2]. We sliced the brightness distribution perpendicular at a fixed PA of $-135^{\circ}$ and measured a full-width half-maximum (FWHM) width of the jet with Gaussian fitting. The measured FWHM width was de-convolved with the size of the restoring beam, thus removing its effect on the measured width.

On the other hand, on VLBI scales, the jet is known to have a complex bending structure ${ }^{2}$. In addition, the transverse structure is highly asymmetric, particularly on 10-mas scales (e.g., [11]). To measure the width of the bending jet on milliarcsecond scales, we adopted the following methods. First, we created a PA profile of the jet brightness by slicing the images with concentric circles centered on the jet core. We fitted circular slices of the brightness distribution with an asymmetric Gaussian with two different half-width-at-half-maximum (HWHM) angles on each side of the peak in order to trace asymmetry in the transverse structure. We adopted the central PA of this HWHM region as the PA of the jet at each distance from the core. Then, we measured the jet width perpendicular to the measured PA at each distance by the asymmetric Gaussian. We de-convolved the average of the best-fit FWHM widths with the size of the restoring beam in the same way as for the arcsecond-scale structure.

Finally, we adopted the de-convolved FWHM width as the final product, satisfying two conditions: (i) larger than the restoring beam (i.e., the transverse structure was resolved enough) and (ii) the best-fit peak intensity of the Gaussian exceeds $20 \sigma$ r.m.s. level of the off-jet brightness distribution.

\section{Results and Discussion}

Figure 1 shows our preliminary results of the jet-width measurements of 3C 273 on scales ranging from sub-pc to kpc. Here, we summarize the global jet structure of 3C 273 from large scales. The jet is terminated in a kpc-scale radio lobe at $\sim 1$ arcsec from the core traced with MERLIN 1.6 GHz data. The jet position angle is stable around $\sim 135^{\circ}$, and the preliminary power-law fits to this region suggest a power-law profile of $r \propto z^{1.17 \pm 0.17}$, where errors are $1 \sigma$, being consistent with a conical flow with an index of unity at the $1 \sigma$ lower-limit. This conical streamline and the position angle profile of the kpc-scale jet is smoothly connected to the profile of the inner very long baseline interferometry (VLBI)-scale jet at $\sim 10-100$ mas traced with VLBA at $1.4 \mathrm{GHz}$ and downstream of the jet traced with the VLBA at $15 \mathrm{GHz}$.

The jet structure starts to dynamically change in the inner $\sim 10$ mas traced with VLBA $15 \mathrm{GHz}$ data. The jet starts to bend slightly in the counter-clockwise direction, with rapid changes in the jet width around $\sim 1$ mas. Beyond the inner $\sim 4$ mas from the core traced with $43 \mathrm{GHz}$ data, the jet propagates at a relatively stable PA in the range of $\sim-140^{\circ}-150^{\circ}$. The opening angle increases for the inner jet at this region, suggesting that the jet is actively collimating. Indeed, the preliminary best-fit power-law model is $r \propto z^{0.47 \pm 0.01}$ for this region, suggesting that the jet has a mostly parabolic shape.

In summary, the jet streamline of 3C 273 has a parabolic shape in the inner 4 mas from the core, while it has a conical shape in the more outer regions until the jet terminates at the kpc lobe. This is the first observational evidence to detect a transition of the streamline from parabolic to conical in a quasar jet. In Figure 2, we compare the streamline profiles of our measurements on 3C 273 and M87. It is notable that both sources have quite similar collimation profiles despite a huge difference in the accretion rates of the central SMBHs expected between the Quasar 3C 273 and the low-luminosity AGN M87. The similarity suggests universality of jet collimation among AGNs with different accretion rates of the central SMBHs. 


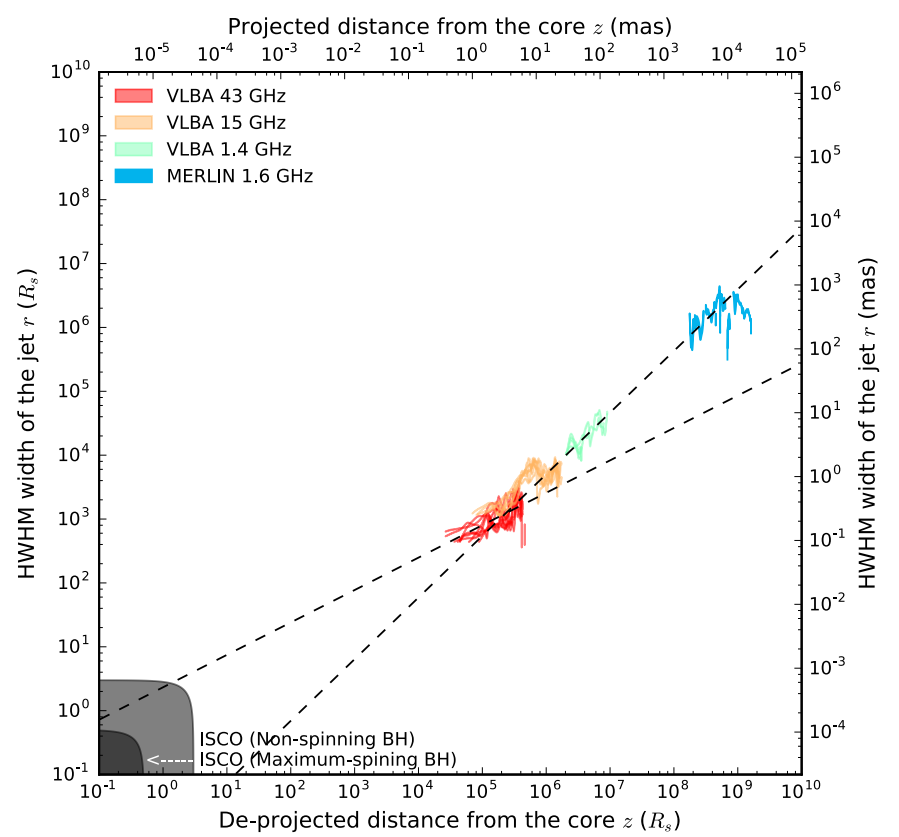

Figure 1. Preliminary results of the measured radius of the jet as a function of the de-projected distance from the core in units of $R_{s}$. The red, orange, and green regions are $1 \sigma$-credible region of the jet width at 43,15 , and $1.4 \mathrm{GHz}$ measured accordingly with Very Long Baseline Array (VLBA) observations. The blue lines are the jet width derived from Multi-Element Radio Linked Interferometer Network (MERLIN) 1.6 GHz data. The jet is described by two different shapes. The shallower dashed line indicates a parabolic structure with a power-law index a of $0.47 \pm 0.01$, where errors are $1 \sigma$ derived from $43 \mathrm{GHz}$ data, while the steeper dashed line indicates a conical structure with an index of $1.17 \pm 0.17$ derived from the kilo-parsec-scale jet traced with VLA observations. The light gray area indicates the radius of the innermost stable circular orbit (ISCO) for a non-spinning black hole (BH), while the darker area indicates the radius of ISCO for the maximum spin with a prograde orbit.

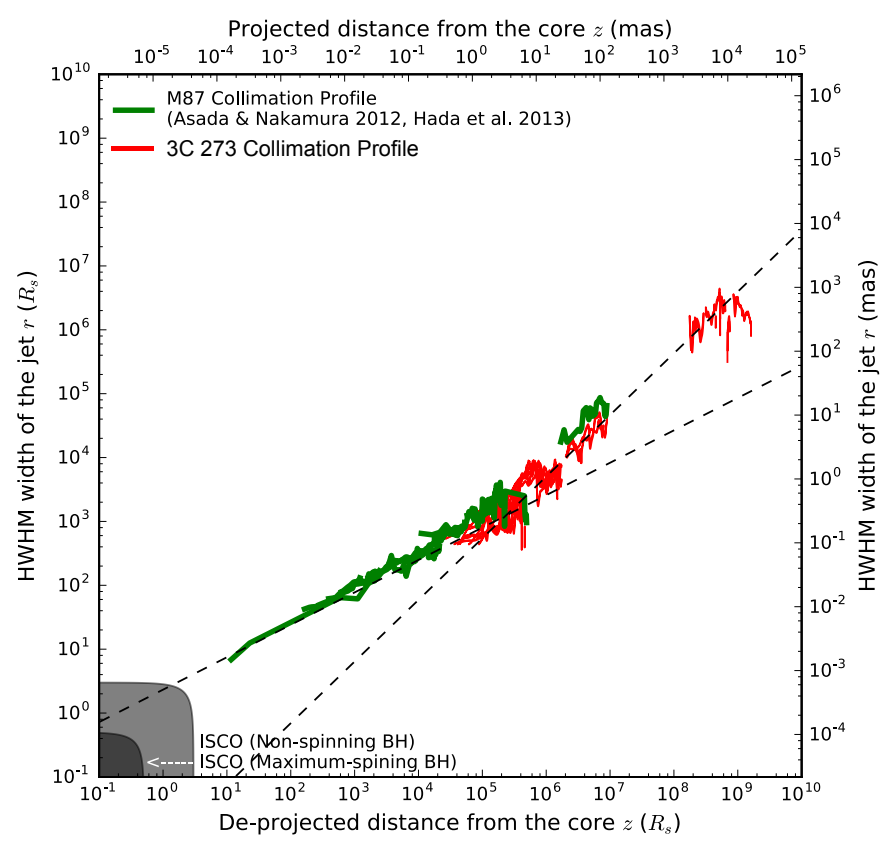

Figure 2. Comparison of the jet-width profiles between M87 [2,3] (green) and our preliminary 3C 273 results (red). 
Current theoretical paradigms for jet formation are based on magnetohydrodynamic (MHD) schemes, where the magnetic field plays an important role in collimating and accelerating the jets $[15,16]$. In the framework of MHD schemes, the differential rotation in the black hole's ergosphere [17] or the inner accretion disk [18] creates a tightly-twisted magnetic field. In the classical picture, the jet collimation is produced by magnetic hoop stresses associated with toroidal field lines [18]. However, recent theoretical studies suggest that external pressure is necessary at the jet boundary, because the magnetic hoop stress is not strong enough to collimate the jet globally in the relativistic regime (e.g., [19]). Recent numerical studies support the need for an external confining pressure (e.g., $[19,20])$.

For M87 whose bolometric luminosity is $\sim 10^{-6}$ times smaller than the Eddington luminosity ( $L_{\text {edd }}$ ) [21], Asada and Nakamura [2] suggest that the jet may be confined by a radiatively inefficient hot accretion flow (RIAF) with a geometrically-thick quasi-spherical shape (e.g., [22]). Indeed, recent $86 \mathrm{GHz}$ observations of M87 have shown that the jet pressure at the $86 \mathrm{GHz}$ core is comparable to the RIAF pressure, indicating that the pressure support from the hot accretion flow may be capable of shaping the M87 jet [5].

On the other hand, 3C 273 has a much higher bolometric luminosity of $\sim 0.13-0.53 L_{\text {edd }}$ [9], implying that the source is driven by an accretion flow with a much higher accretion rate, such as for a standard thin disk [23]. Given the geometrically-thin shape expected for this regime of the black-hole accretion flow, the streamline of 3C 273 suggests that the material confining the jet would be different from other low luminosity AGNs, and simultaneously poses a question about how the circum-jet material has enough external pressure to support jet confinement in luminous AGNs with quite different accretion regimes. Considering that the radiation pressure from the central accretion flow plays an important role in the hydrodynamics of such high-Eddington-ratio sources as 3C 273, future radiative MHD work will be essential to understand the presented collimation profile of 3C 273. We note that future multi-scale Faraday rotation measurements of jets through multi-frequency polarimetry would be useful to diagnose the profile of the magnetized circum-jet materials and its relation to the jet collimation.

\section{Summary}

We have presented our preliminary results of multi-frequency VLBI observations of 3C 273 with the VLBA and MERLIN. With its global jet structure on a wide range of scales from sub-pc to $\mathrm{kpc}$, we discovered a transition from a parabolic stream to a conical stream, which is seen in the much lower-powered radio galaxies M87 and NGC 6251. The similarity in the profile to jets in such lower-powered AGNs indicate that the jet collimation may have universality among the AGNs with different accretion rates of the central SMBH. Simultaneously, our results suggest that quasar jets have circum-jet material in which gas pressure is enough to support jet confinements and collimation, which may have a different origin compared with low-luminosity AGNs.

Future work will focus on the innermost region where the jet is actively collimated. We will also reduce data at other wavelengths complementing our current profile of the jet collimation and also the circum-jet material traced with Faraday rotation. We recently conducted ultra-high-angular VLBI observations with the Global Millimeter VLBI Array (GMVA) at $86 \mathrm{GHz}$ and the Event Horizon Telescope (EHT) at $230 \mathrm{GHz}$, including the Atacama Large Millimeter/submillimeter Array (ALMA). Our on-going and future studies with new high-quality full-polarization data will provide a more detailed picture of jet collimation in a quasar jet.

Acknowledgments: Kazunori Akiyama and this work are financially supported by grants from the National Science Foundation (AST-1440254, AST-1614868) and by the Jansky Fellowship of the National Radio Astronomy Observatory (NRAO). This study makes use of $43 \mathrm{GHz}$ VLBA data from the VLBA-BU Blazar Monitoring Program (VLBA-BU-BLAZAR; http:/ / www.bu.edu/blazars/VLBAproject.html), funded by NASA through the Fermi Guest Investigator Program. The VLBA is an instrument of the National Radio Astronomy Observatory. The National Radio Astronomy Observatory is a facility of the National Science Foundation operated by Associated Universities, Inc. This research also has made use of data from the MOJAVE database that is maintained by the 
MOJAVE team [14]. MERLIN is a National Facility operated by the University of Manchester at Jodrell Bank Observatory on behalf of STFC.

Author Contributions: Kazunori Akiyama designed the study and leaded all the process of this work, which include analysis and interpretation of data, and writing the draft of the manuscript. All other authors have contributed to discussions on all the processes of this work, and critically reviewed the manuscript.

Conflicts of Interest: The authors declare no conflict of interest.

\section{References}

1. Yuan, F.; Narayan, R. Hot Accretion Flows Around Black Holes. Annu. Rev. Astron. Astrophys. 2014, 52, 529-588.

2. Asada, K.; Nakamura, M. The Structure of the M87 Jet: A Transition from Parabolic to Conical Streamlines. Astrophys. J. Lett. 2012, 745, L28.

3. Hada, K.; Kino, M.; Doi, A.; Nagai, H.; Honma, M.; Hagiwara, Y.; Giroletti, M.; Giovannini, G.; Kawaguchi, N. The Innermost Collimation Structure of the M87 Jet Down to 10 Schwarzschild Radii. Astrophys. J. 2013, 775,70 .

4. Nakamura, M.; Asada, K. The Parabolic Jet Structure in M87 as a Magnetohydrodynamic Nozzle. Astrophys. J. 2013, 775, 118.

5. Hada, K.; Kino, M.; Doi, A.; Nagai, H.; Honma, M.; Akiyama, K.; Tazaki, F.; Lico, R.; Giroletti, M.; Giovannini, G.; et al. High-sensitivity $86 \mathrm{GHz}(3.5 \mathrm{~mm})$ VLBI Observations of M87: Deep Imaging of the Jet Base at a Resolution of 10 Schwarzschild Radii. Astrophys. J. 2016, 817, 131-147.

6. Tseng, C.Y.; Asada, K.; Nakamura, M.; Pu, H.Y.; Algaba, J.C.; Lo, W.P. Structural Transition in the NGC 6251 Jet: An Interplay with the Supermassive Black Hole and Its Host Galaxy. Astrophys. J. 2016, 833, 288.

7. Nagai, H.; Haga, T.; Giovannini, G.; Doi, A.; Orienti, M.; D’Ammando, F.; Kino, M.; Nakamura, M.; Asada, K.; Hada, K.; et al. Limb-brightened Jet of 3C 84 Revealed by the $43 \mathrm{GHz}$ Very-Long-Baseline-Array Observation. Astrophys. J. 2014, 785, 53.

8. Boccardi, B.; Krichbaum, T.; Bach, U.; Ros, E.; Zensus, J.A. High resolution mm-VLBI imaging of Cygnus A. arXiv 2015, arXiv:astro-ph.HE/1504.01272.

9. Paltani, S.; Türler, M. The mass of the black hole in 3C 273. Astron. Astrophys. 2005, 435, 811-820.

10. Bahcall, J.N.; Kirhakos, S.; Saxe, D.H.; Schneider, D.P. Hubble Space Telescope Images of a Sample of 20 Nearby Luminous Quasars. Astrophys. J. 1997, 479, 642-658.

11. Lobanov, A.P.; Zensus, J.A. A Cosmic Double Helix in the Archetypical Quasar 3C273. Science 2001, 294, 128-131.

12. Meyer, E.T.; Sparks, W.B.; Georganopoulos, M.; Anderson, J.; van der Marel, R.; Biretta, J.; Sohn, S.T.; Chiaberge, M.; Perlman, E.; Norman, C. An HST Proper-motion Study of the Large-scale Jet of 3C273. Astrophys. J. 2016, 818, 195.

13. Shepherd, M.C.; Pearson, T.J.; Taylor, G.B. DIFMAP: An Interactive Program for Synthesis Imaging. Bull. Am. Astron. Soc. 1994, 26, 987-989.

14. Lister, M.L.; Cohen, M.H.; Homan, D.C.; Kadler, M.; Kellermann, K.I.; Kovalev, Y.Y.; Ros, E.; Savolainen, T.; Zensus, J.A. MOJAVE: Monitoring of Jets in Active Galactic Nuclei with VLBA Experiments. VI. Kinematics Analysis of a Complete Sample of Blazar Jets. Astron. J. 2009, 138, 1874-1892.

15. Lovelace, R.V.E. Dynamo model of double radio sources. Nature 1976, 262, 649-652.

16. Blandford, R.D. Accretion disc electrodynamics-A model for double radio sources. Mon. Not. R. Astron. Soc. 1976, 176, 465-481.

17. Blandford, R.D.; Znajek, R.L. Electromagnetic extraction of energy from Kerr black holes. Mon. Not. R. Astron. Soc. 1977, 179, 433-456.

18. Blandford, R.D.; Payne, D.G. Hydromagnetic flows from accretion discs and the production of radio jets. Mon. Not. R. Astron. Soc. 1982, 199, 883-903.

19. Komissarov, S.S.; Barkov, M.V.; Vlahakis, N.; Königl, A. Magnetic acceleration of relativistic active galactic nucleus jets. Mon. Not. R. Astron. Soc. 2007, 380, 51-70.

20. Nakamura, M.; Li, H.; Li, S. Structure of Magnetic Tower Jets in Stratified Atmospheres. Astrophys. J. 2006, $652,1059-1067$. 
21. Prieto, M.A.; Fernández-Ontiveros, J.A.; Markoff, S.; Espada, D.; González-Martín, O. The central parsecs of M87: Jet emission and an elusive accretion disc. Mon. Not. R. Astron. Soc. 2016, 457, 3801-3816.

22. Narayan, R.; Fabian, A.C. Bondi flow from a slowly rotating hot atmosphere. Mon. Not. R. Astron. Soc. 2011, $415,3721-3730$.

23. Shakura, N.I.; Sunyaev, R.A. Black holes in binary systems. Observational appearance. Astron. Astrophys. 1973, 24, 337-355.

(C) 2018 by the authors. Licensee MDPI, Basel, Switzerland. This article is an open access article distributed under the terms and conditions of the Creative Commons Attribution (CC BY) license (http://creativecommons.org/licenses/by/4.0/). 